\title{
Oportunidades para acercar la ciencia a la práctica de la restauración de bosques y arbustales de Polylepis
}

\author{
Laura V. Morales ${ }^{1, \bigotimes}$; Beatriz Fuentealba ${ }^{2} ;$ C. Steven Sevillano $^{3} ;$ M. Isabel \\ Gómez; M. Claudia Segovia-Salcedo ${ }^{5}$; Daniel Renison ${ }^{6}$; Daniel Green; ; \\ Constantino Aucca ${ }^{8}$ \& Isabell Hensen ${ }^{9,10}$
}

\begin{abstract}
${ }^{1}$ Department of Plant Sciences, University of California, Davis, CA, United States of America. ${ }^{2}$ Instituto de Montaña, Huaraz, Ancash, Perú. ${ }^{3}$ Cornell Lab of Ornithology, Ithaca, NY, United States of America. ${ }^{4}$ Sección de Ornitología, Colección Boliviana de Fauna, Museo Nacional de Historia Natural, La Paz, Bolivia. ${ }^{5}$ Departamento de Ciencias de la Vida y Agricultura, Universidad de las Fuerzas Armadas- ESPE, Sangolquí, Ecuador. ' Instituto de Investigaciones Biológicas y Tecnológicas, Centro de Ecología y Recursos Naturales Renovables, Dr. Ricardo Luti. CONICET - Universidad Nacional de Córdoba, Córdoba, Argentina. ${ }^{7}$ Meristema Consultores, Santiago, Chile. ${ }^{8}$ Asociación Ecosistemas Andinos ECOAN, Cusco, Cusco, Perú. ${ }^{9}$ Institute of Biology/Geobotany and Botanical Garden, Martin Luther University of Halle-Wittenberg, Halle/Saale, Germany. ${ }^{10}$ German Centre for Integrative Biodiversity Research (iDiv) Halle-Jena-Lepzig, Leipzig, Germany.
\end{abstract}

\begin{abstract}
Resumen. ¿Cómo acercar la ciencia a la práctica de la restauración de los bosques de Polylepis? Esta pregunta suscitó la discusión y la reflexión entre un grupo de científicos académicos y practicantes de restauración que participamos del simposio "Reforestación y Restauración de Ecosistemas de Polylepis: Experiencias y Perspectivas" durante el IV Congreso Internacional de Ecología y Conservación de Bosques de Polylepis. El diálogo dentro del grupo nos permitió reconocer que existen diferentes experiencias prácticas relacionadas con la reforestación con especies de Polylepis, con distintos objetivos, grado de avance y de éxito, y, en general, poca participación del sector académico. Para que los científicos colaboremos de una manera más efectiva con la restauración de estos bosques, tenemos que realizar investigaciones tanto en áreas relevantes para la ciencia como para la práctica, y compartir nuestros aportes con los practicantes. En este artículo identificamos temas que consideramos prioritarios como guía para definir el marco de trabajo de investigación en la restauración de los bosques de Polylepis y los presentamos de acuerdo con su importancia para los sectores académicos y de practicantes. El sector académico considera que los principales vacíos de información en los que hay que trabajar se relacionan con aspectos ecológicos que abarcan desde la escala genética hasta la de paisaje. Mientras tanto, el sector de los practicantes sostiene que es necesario priorizar aspectos técnicos aplicables a su labor, como selección del sitio, manejo de las especies en vivero y en campo, e identificación de las barreras al establecimiento. Finalmente, sugerimos algunas estrategias de comunicación y cooperación directa en investigación para facilitar la colaboración entre científicos y practicantes de restauración, y para lograr juntos el objetivo de conservar y restaurar los ecosistemas de Polylepis.
\end{abstract}

[Palabras clave: reforestación, bosques andinos, Congreso Jujuy 2016, estrategias de cooperación]

\begin{abstract}
Aвstract. Opportunities to bridge gaps between science and practice in restoration of Polylepis ecosystems. How do we bridge the gaps between science and practice in the restoration of Polylepis forests and shrublands? This question inspired discussion and reflection among a group of academic scientists and restoration practitioners attending the "Reforestation and Restoration in Polylepis Ecosystems: Experiences and Perspectives" symposium held during the IV International Congress on Ecology and Conservation of Polylepis Forests. Through dialogue, we recognized there are different experiences related to reforestation with Polylepis trees/shrubs, each with different objectives and degrees of progress and success, and, in general, very low participation of the academic sector. In order for academic scientists to contribute more effectively towards the restoration of these forests, we need to research areas of relevance both to science and restoration practice and explicitly share the implications of our research with practitioners. Here, we seek to guide the future research agenda in Polylepis forest restoration and reforestation science by identifying topics that should have priority in future studies, and present them according to the priorities of academics and practitioners. While the academic sector considers main gaps in knowledge requiring work to be related to ecological aspects at several scales ranging from genetics to landscape, restoration practitioners prioritize research of technical aspects directly applicable to their work (e.g., site selection, species management in greenhouses and the field, identification of barriers to establishment). Finally, we make some suggestions about research communication and direct collaboration between scientists and restoration practitioners to help us accomplish the main objective of conserving and restoring Polylepis ecosystems together.
\end{abstract}

[Keywords: reforestation, Andean forests, Jujuy 2016 Conference, cooperative strategies]

Editora asociada: Erica Cuyckens

\1vmorales@ucdavis.edu
Recibido: 13 de febrero de 2017

Aceptado: 22 de septiembre de 2017 


\section{INTRODUCCIÓN}

La restauración ecológica, entendida como una actividad intencional que inicia o acelera la recuperación de un ecosistema que fue degradado, dañado o alterado (SER 2004), requiere de la participación tanto del sector académico como de aquellos que llevan a cabo la práctica y la implementación técnica a campo (i.e., comitéslocales, agenciasgubernamentales, ONG y consultores privados [denominados de aquí en adelante "practicantes"]). Sin embargo, en el caso de la restauración de bosques y arbustales de Polylepis, los académicos y los practicantes generalmente actuamos de forma independiente. Los proyectos de reforestación son desarrollados por practicantes, con poca intervención de los académicos (e.g., Fuentealba and Sevillano 2016), las experiencias y necesidades de los practicantes son poco consideradas en el sector académico, $\mathrm{y}$, en general, los resultados de las investigaciones pocas veces se convierten en sugerencias para mejorar las prácticas de campo. La colaboración escasa entre practicantes y científicos es recurrente en todo el mundo y tipos de ecosistemas (e.g., Palmer 2009; Cabin et al. 2010); esto evita que se construya y se avance, en conjunto, hacia el objetivo común de conservar y restaurar los ecosistemas.

Tomando en cuenta esto, el 8 de septiembre de 2016 desarrollamos el simposio "Reforestación y Restauración de Ecosistemas de Polylepis: Experiencias y Perspectivas" dentro del IV Congreso de Ecología y Conservación de Bosques de Polylepis (Jujuy, Argentina). El simposio contó con la participación de varios expertos, académicos y practicantes de 7 países (con experiencia en todos los países andinos excepto Venezuela y Colombia), que compartimos nuestras experiencias en proyectos de restauración con Polylepis o investigaciones afines con el objetivo de reflexionar y encontrar soluciones que ayuden a acercar la academia a la práctica de restauración y conservación de los bosques de Polylepis, y viceversa.

Posterior al simposio, los autores discutimos las formas en que los científicos podemos colaborar de manera más activa y efectiva en la restauración de los bosques de Polylepis a través de la investigación. Discutimos algunos vacíos de información ecológica cuya investigación ayudaría a generar proyectos de reforestación y restauración más sostenibles. Descubrimos que los temas de investigación prioritarios para el sector académico no necesariamente son los mismos que para los practicantes, aunque pueden estar relacionados, y que las prioridades pueden cambiar con las circunstancias locales. Aunque reconocemos que existe una cantidad de temas posibles y diversidad entre los países, aquí presentamos los temas que priorizamos los autores desde nuestra experiencia. Esperamos que sirvan de guía en el marco de trabajo para la restauración de estos bosques. Además, sugerimos algunas estrategias para mejorar la comunicación de resultados y la cooperación directa entre científicos académicos y practicantes de restauración.

\section{El CONTEXTO ACTUAL PARA LA Restauración de Polylepis}

En la actualidad, los bosques y arbustales dominados por Polylepis tienen una distribución disyunta a lo largo de los Andes y las Sierras de Córdoba (Argentina), donde se encuentran como poblaciones aisladas de árboles o arbustos de Polylepis, presentes en parches de distintos tamaños y densidades entre un paisaje de pastizal de altura (Simpson 1979; Kessler 2006; ver Sevillano-Ríos et al. en este número, Figura 1). Aunque es un tema de debate (Renison et al. en este número), es probable que esta distribución en parches se deba a una combinación de factores naturales (e.g., cambios climáticos pasados [Rangel and Arellano 2010], distribución heterogénea de microhábitats en topografías montañosas [Rada 2009; Ashcroft and Gollan 2013] y fragmentación por los seres humanos [Purcell and Brelsford 2004]). Dada la amplia distribución latitudinal de estos bosques y las diferencias locales en la historia climática y de uso de suelo, es posible que la influencia relativa de las distintas clases de factores varíe según la zona. Sin embargo, hoy día, estos bosques sufren una amenaza constante debido a diferentes actividades humanas (e.g., extracción de leña, quema intencional, construcción de infraestructura, forestación con especies exóticas y actividades industriales que aumentan la concentración de gases de efecto invernadero y que contribuyen al cambio climático [Navarro et al. 2010; Renison et al. 2013; Cuyckens et al. 2016; Fuentealba and Sevillano 2016]). Estos ecosistemas son reconocidos como centros de endemismo de alta diversidad biológica y por brindar servicios ecosistémicos clave a las poblaciones de montaña (Fjeldsa 2002; Gareca et al. 2010). Por ello, el interés por su 
Ciencia y práctica de la Restauración en Polylepis

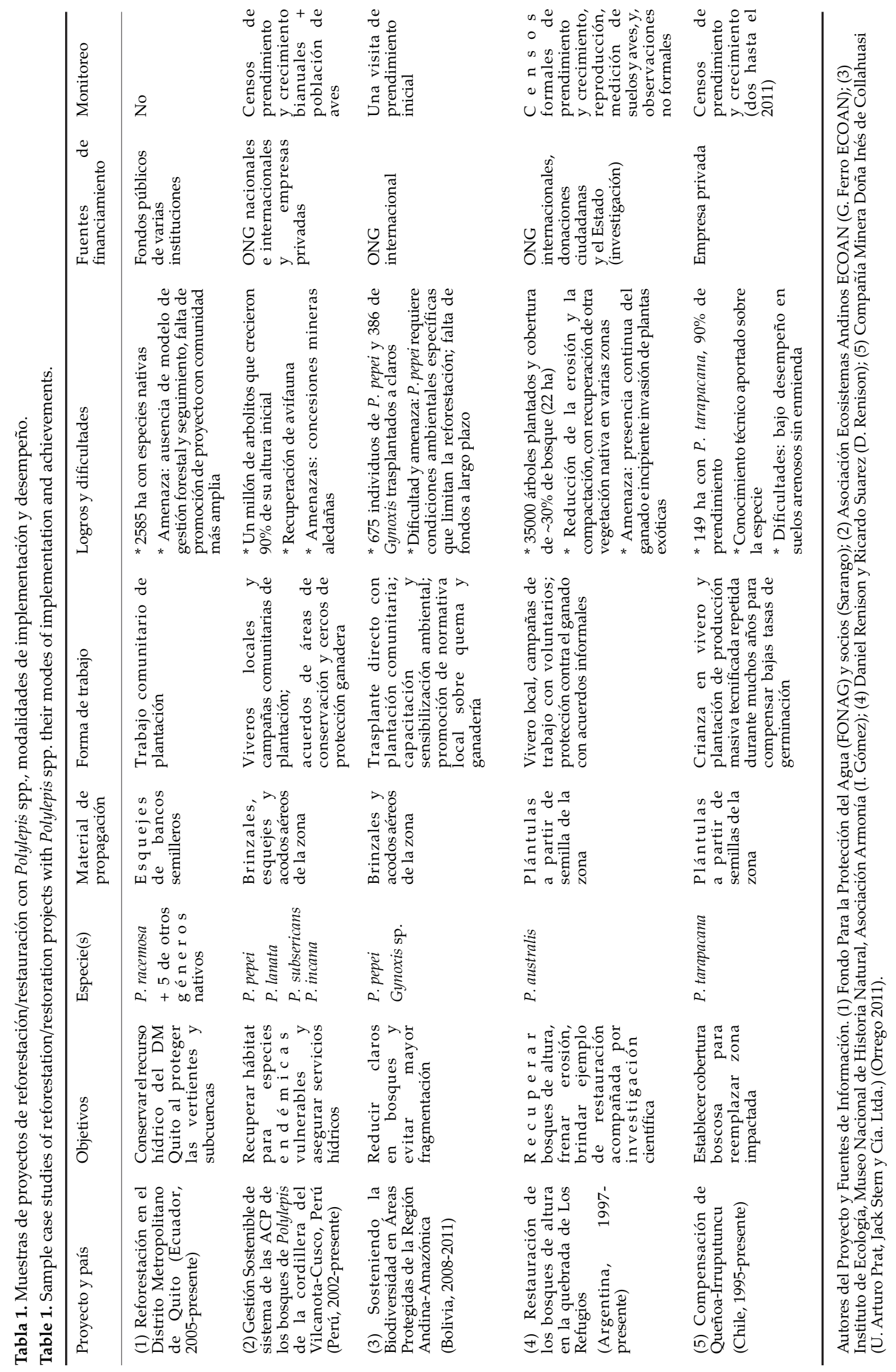


conservación aumentó entre las instituciones públicas y privadas, que promovieron la creación de áreas naturales protegidas y que desarrollan proyectos de reforestación. En algunos países, como Chile, se promulgaron leyes que exigen acciones compensatorias cuando estas comunidades vegetales son afectadas.

Dentro de las experiencias de restauración que revisamos, encontramos una variedad de proyectos con diferentes grados de avance, conocimiento e información disponible, según el país y la especie de Polylepis (Tabla 1). Por ejemplo, dispone de menos información para algunas especies (e.g., P. pacensis), mientras que los mayores avances en el conocimiento sobre el manejo de la propagación y el establecimiento de especies nativas se encuentran en la Argentina y en Chile para $P$. australis y $P$. tarapacana, respectivamente. También observamos que los practicantes generaron técnicas y métodos propios para cumplir sus objetivos de reforestación con poco apoyo del sector académico. Los proyectos de restauración varían en costos y financiamiento desde pequeños proyectos con bajo presupuesto, que dependen del apoyo de comunidades locales o voluntarios (e.g., Renison et al. 2016, Tabla 1, Proyecto 3) hasta grandes proyectos, financiados por compañías privadas que implementan propagación y manejo tecnificado de las plantaciones (e.g., Orrego 2011, Tabla 1, Proyecto 4). Los objetivos, los niveles de éxito, el monitoreo y las dificultades de estos proyectos también cambian, puesto que algunos están centrados sólo en establecer árboles de Polylepis, mientras que otros buscan, además, aumentar la biodiversidad y los servicios ecosistémicos, generar beneficios para la población local y hasta promover la investigación científica en restauración.

A pesar de la diversidad de proyectos de reforestación y restauración, identificamos algunos temas de investigación que se deberían atender de manera prioritaria en los siguientes años a fin de promover que sean más sostenibles. Una preocupación común entre los sectores académicos y practicantes es reconocer el posible impacto que tendrá el cambio climático sobre la distribución y la conservación de los bosques de Polylepis, así como sus efectos sobre las áreas ya reforestadas. Sin embargo, los dos sectores priorizan de distintas formas otros temas importantes. A continuación, presentamos esos temas.

\section{Prioridades PARA EL SECTOR ACADÉMICO}

\section{Aspectos genéticos en el manejo de las especies del género Polylepis}

Las prácticas de restauración pueden afectar la salud genética de poblaciones amenazadas; este es un aspecto que puede limitar su capacidad de persistir en el tiempo (Falk et al. 2006). De acuerdo con observaciones en el campo, aunque varias especies de Polylepis se encuentren en la misma localidad, frecuentemente se presentan en parches dominados por sólo una especie del género (L. Morales, observación personal; Kessler 2006). Asimismo, investigaciones recientes mostraron que Polylepis presenta poliploidías (i.e., tiene más de dos juegos de cromosomas completos) y una capacidad de hibridación elevada (Schmidt-Lebuhn et al. 2006; SegoviaSalcedo et al. 2011; Segovia-Salcedo and Quijia-Lamiña 2014). Por ello, se considera que utilizar especies de Polylepis que no sean locales en los proyectos de reforestación es un riesgo para la diversidad genética de las poblaciones naturales. Conocemos poco de las consecuencias que genera la hibridación entre especies, porque los problemas en el proceso de adaptación de nuevas especies o genotipos introducidos en una zona generalmente se manifiestan a mediano y largo plazo o durante la ocurrencia de eventos ambientales extremos (Thomas et al. 2014). No obstante, los efectos que podrían estar teniendo lugar son la generación de descendencia con menor vigor, menor adaptación al medio, menor capacidad reproductiva o mayor vigor y competitividad de híbridos versus las especies de padres. Todos estos son escenarios que aumentan la vulnerabilidad de las poblaciones originales (Rhymer and Simberloff 1996; Segovia-Salcedo 2011; Segovia-Salcedo et al. 2011).

Entender mejor este proceso ayudaría a recomendar cómo mantener o incrementar la diversidad genética de las poblaciones naturales. Por ahora, para evitar estos riegos se sugiere usar material de propagación de procedencia local. Estos cuidados son más importantes en las zonas que presentan una mayor diversidad de especies, como son los Andes Centrales (Bolivia, Perú, Ecuador), ya que la identificación taxonómica en campo es difícil. Ya se introdujo la especie $P$. racemosa desde Perú hacia Ecuador para usarla en proyectos de forestación (Segovia-Salcedo et al. 2011). También se encontraron en 
Ecuador zonas de plantación con $P$. reticulata cercanas a bosques naturales dominados por $P$. incana y P. pauta. De forma similar, en Perú se plantaron $P$. racemosa y $P$. incana en áreas cercanas a bosques de $P$. sericea y $P$. weberbaueri (Fuentealba and Sevillano 2016).

Otra práctica que puede estar afectando la diversidad genética en las plantaciones de Polylepis es el uso de plantas propagadas vegetativamente (i.e., esquejes, acodos), en lugar de semillas. La propagación vegetativa es una alternativa muy útil ante las bajas tasas de germinación típicas del género y la alta mortalidad inicial de las plántulas. Además, esta manera de propagación permite producir más individuos, más rápido y con técnicas estandarizadas, fáciles de implementar con personal voluntario o con agricultores locales. Sin embargo, en $P$. australis se determinó que el crecimiento era más lento en los árboles producidos por esqueje, en comparación con los producidos a partir de semillas (Renison and Cingolani 1998). Además, si no se toma la precaución de usar diferentes árboles semilleros y de procedencia local, se puede reducir la diversidad genética y crear problemas ecológicos como el aumento de la endogamia y la mayor susceptibilidad ante cualquier plaga, enfermedad o evento climático adverso (Thomas et al. 2014). En este caso, tampoco sabemos si esta práctica tiene consecuencias sobre la capacidad reproductiva de los individuos plantados, lo que afectaría la capacidad de regeneración en los bosques reforestados.

\section{Conocimiento auto-ecológico de Polylepis}

Algunos aspectos del conocimiento autoecológico son básicos para el manejo de las poblaciones naturales. Sin embargo, este conocimiento es limitado para la mayoría de las especies de Polylepis. Incluso, aunque la ecología de poblaciones es el tema con mayor cantidad de estudios publicados, el conocimiento está agregado en pocas especies y regiones (Renison et al. en este número). En particular, es importante conocer mejor la dinámica poblacional reproductiva (incluyendo formación de bancos de semillas o prevalencia de reproducción vegetativa). Los estudios sobre la supervivencia, el desempeño y los factores que afectan la población juvenil sondemuchautilidad tantoparala restauración como para la conservación y aportan a temas prioritarios para los practicantes. Otro tema básico para la restauración es el conocimiento del nicho ecológico de las distintas especies en sus distintos estadíos, ya que pueden aportar a la elección de especies y sitios de reforestación, como describiremos más adelante al presentar las prioridades de los practicantes.

\section{Ecología de ecosistemas y comunidades en bosques de Polylepis}

Para promover actividades que no sólo busquen recuperar la cobertura de Polylepis, sino la restauración ecológica del ecosistema, es importante conocer mejor la estructura, la composición, las interacciones de los diversos habitantes y las funciones ecológicas en los bosques remanentes. Si se considera la amplia distribución geográfica de los bosques de Polylepis, resulta poco el conocimiento generado hasta el presente a nivel de ecosistema. Aunque la biodiversidad y las comunidades asociadas es un tema de estudio muy popular (ver Renison et al. en este número), para muchas regiones se tiene poca información de la biota asociada, más allá de la avifauna (e.g., artrópodos, especies leñosas acompañantes).Muchadeestainformaciónestá en forma de inventarios (e.g., listas de especies, asociaciones fitosociológicas). Más escasa aun es la información ecológica de estas especies y de las interacciones y funciones ecológicas entre los distintos componentes abióticos y bióticos que mantienen estos ecosistemas. En particular, hay un gran vacío en el conocimiento sobre las funciones ecosistémicas de estos bosques, aunque su recuperación sea uno de los objetivos de algunos proyectos de restauración (e.g., Sarango). Sólo conociendo mejor estas funciones y las interacciones que las sostienen se podrán dar recomendaciones para recuperarlas adecuadamente en las áreas reforestadas.

\section{Ecología del paisaje de los bosques de Polylepis}

En paisajes fragmentados, la conectividad a nivel del paisaje es un tema de trabajo fundamental en proyectos de restauración y conservación a mayores escalas (Rudnick et al. 2012). Sin embargo, este es un tema relativamente nuevo y poco estudiado en Polylepis (e.g., revisión genética en Gareca et al. 2013, dispersión de semillas y polen; Torres et al. 2008; Seltmann et al. 2009). Esto incluye reconocer, entre otros temas y para las diferentes especies de Polylepis, cómo se facilita la conectividad del paisaje y el flujo génico entre parches, y cuáles son las principales barreras para este flujo. Es importante que estos estudios se repitan en 
diferentes especies y sitios, ya que pueden existir patrones contrastantes, como lo demostraron algunos estudios acerca del flujo génico y la conectividad genética a nivel de paisaje en $P$. australis (Argentina, alto flujo génico y conectividad; Hensen et al. 2011) y P. incana (Ecuador, bajo flujo génico y conectividad; Hensen et al. 2012). Contar con más conocimiento nos permitirá dar recomendaciones para un mejor manejo de los relictos de bosque y de la matriz que los rodea, y para seleccionar sitios de reforestación que ayuden a mantener o a recuperar la conectividad del paisaje. En algunos casos (aunque no en todos) (e.g., Peng et al. 2017), los mismos estudios de conectividad genética pueden brindar información que ayuda a distinguir la antigüedad (histórica vs. reciente) y, por ende, la causa (natural vs. antrópica) del actual estado fragmentado de los bosques (Gareca et al. 2013).

\section{PRIORIDADES PARA LOS PRACTICANTES}

Uno de los objetivos de los practicantes es el de maximizar la superviviencia y el desempeño de los individuos de Polylepis plantados. Las prioridades más inmediatas requieren de estudios que respondan dónde y cómo alcanzar este objetivo.

\section{Selección de la zona y de las especies para reforestar}

Para cualquier reforestación, la selección de las zonas y las especies para establecer las plantaciones se debe considerar con seriedad debido a la inversión que implica un proyecto de reforestación a largo plazo. En la restauración de bosques de Polylepis, la selección de zonas a nivel local en el paisaje siempre ha sido problemática. En las zonas adyacentes a los parches remanentes, la decisión es relativamente sencilla, e incluso puede resultar menos costoso favorecer el establecimiento natural de las plántulas y los renovales en zonas aledañas a parches, donde estarían protegidas del disturbio antrópico, por ejemplo. Pero en las áreas con ausencia local de Polylepis, la decisión es más difícil. No siempre se tiene evidencia de su existencia histórica y no se sabe si esa ausencia se debe a características ambientales inadecuadas o a la acción del hombre.

En este caso, se podrían elegir áreas con condiciones ambientales similares a las zonas donde hay bosques, asumiendo que la ausencia del bosque en ese lugar se debe a causas antrópicas; esto se debería hacer con buenos criterios. Para generar estos criterios se necesita comprender el nicho ecológico de las distintas especies de Polylepis. Conocer mejor las tolerancias y los requerimientos ecológicos, además de las medidas de desempeño (e.g., tasas de crecimiento) de las diferentes especies ayudará a basar la selección de sitios en la compatibilidad óptima de la especie con las condiciones ambientales del paisaje para lograr las metas dentro del tiempo establecido por el proyecto o, en todo caso, ajustar las expectativas. Para esta tarea pueden ser valiosas más investigaciones ecofisiológicas.

La selección de sitios adecuados depende dela selección de la especie para reforestar. Aunque es preferible usar la especie de Polylepis que se encuentra en el sitio, por varios motivos no siempre es posible, y en otros casos existe más de una especie nativa a la zona que se podría emplear. Pero en todos los casos, es relevante usar una especie adecuada para el sitio. Existen anécdotas acerca del mal desempeño a largo plazo de algunas plantaciones de Polylepis en Perú, donde se sospecha el uso de sitios sub-óptimos o inadecuados para la especie utilizada, en particular donde se usaron especies no locales (C. Aucca, observación personal). Incluso, para ciertas especies que co-ocurren ya hay evidencia de diferencias en la zonificación ecológica por elevación y orientación de las vertientes dentro de una región (Kessler et al. 2014) e, incluso, dentro de un mismo valle (Morales 2017). Esto es un tema para profundizar y estudiar comparativamente en más especies para dar recomendaciones más precisas.

\section{Aspectos de la propagación, el manejo y el establecimiento de las plantas}

Aparte de las consideraciones ecológicas de la selección de especie, los proyectos de reforestación muchas veces enfrentan la decisión de si usar las especies para las cuales hay más información disponible sobre su propagación y manejo (e.g., $P$. racemosa, $P$. australis) o invertir en experimentos y generar una técnica propia para trabajar con especies locales sin información previa. En algunos casos se realizaron pequeñas investigaciones a nivel local, cuyos resultados no están disponibles fuera del instituto en el que se llevaron a cabo. Ante esta situación, se necesita no sólo realizar estudios detallados sobre la reproducción y la propagación por semilla de especies menos conocidas, sino también 
publicarlos. Asimismo, se deberían generar más recomendaciones técnicas específicas, relacionadas con el manejo de las plantas en vivero (e.g., tipo de sustrato, tamaño y forma del contenedor de las plantas, relación óptima entre tamaño radicular y parte aérea de una planta para ser plantada, etc.) y en la plantación (e.g., distancia de plantación, tipo de poda, necesidades de riego y épocas adecuadas de plantación, medidas de protección para prevenir la herbivoría por roedores y artrópodos). Actualmente, existen pocas recomendaciones sobre estos aspectos, aunque se dispone de información general recopilada en manuales agroforestales para algunas especies de Polylepis (e.g., Reynel and Marcelo 2007; Mindreau and Zuñiga 2010). Otro vacío importante es el escaso conocimiento sobre las enfermedades y las plagas que afectan a las distintas especies del género. Generar este conocimiento es fundamental si se quiere promover proyectos de reforestación, a costos razonables, que sean viables y sostenibles, y que usen germoplasma local.

Un aspecto poco conocido es cómo las distintas especies, en sus primeros estadíos, responden a los factores ambientales o de sitio, como el tipo de suelo (según textura o fertilidad), las limitaciones climáticas (falta de agua, heladas, etc.) (e.g., Renison et al. 2005; Rada et al. 2011; Ayma-Romay et al. 2015) y a factores antrópicos como la presencia de ganado, especies exóticas y quemas, etc. (e.g., Gareca et al. 2007; Cierjacks et al. 2008; Renison et al. 2015). Más estudios sobre los factores ambientales y antrópicos que inciden en el éxito de las plantaciones permitirían dar sugerencias a los practicantes sobre cómo reducir la mortalidad y favorecer el establecimiento de cierta especie en campo, según las condiciones del sitio. También ayudarían a manejar mejor la regeneración natural en bosques remanentes como una estrategia de restauración y hasta aprovecharla de forma sostenible como banco natural de plántulas. Por ejemplo, aunque se favorece plantar cerca de bosques remanentes, se han hecho pocos estudios sistemáticos que examinan si los bordes de bosque presentan mejores microclimas y se pueden aprovechar para la expansión natural o asistida de bosques (e.g., Morales 2017). De todos los factores antrópicos, el impacto que produce la ganadería en las áreas reforestadas es uno de los que requiere mayor atención, si consideramos el efecto negativo que se ha observado sobre P. australis en la Argentina (e.g., Renison et al. 2015).

\section{OpORTUNIDADES Y ESTRATEgIAS DE COLABORACIÓN}

Para promover proyectos que nos permitan alcanzar la meta de conservar y restaurar los ecosistemas dominados por Polylepis es necesario llenar los vacíos de información descritos y seguir acercando el trabajo de los académicos al de los practicantes, y viceversa, para construir así grupos multidisciplinarios e iniciativas que permitan una mayor colaboración entre ellos. Pensando en esto, identificamos algunas estrategias que habría que promover para fortalecer el vínculo entre la ciencia y la práctica.

Es importante que haya mayor flujo de información para establecer un diálogo real. El sector académico deberá invertir más tiempo en comprender las necesidades de los practicantes, en difundir los conocimientos adquiridos y en generar recomendaciones para aquellos que implementan el trabajo en campo. Por su parte, el sector de los practicantes deberá invertir tiempo en presentar sus necesidades a los académicos; cuando hayan generado técnicas y métodos propios, podrían divulgarlos fuera de la institución en la que trabajan. En ambos casos, hay pocos incentivos para dedicar tiempo a estas actividades.

Teniendo en cuenta esto, hay que buscar alternativas que permitan organizar, sistematizar y compartir la información generada por ambos sectores en formatos sencillos y a través de diferentes medios de comunicación. Por ejemplo: manuales con recomendaciones para las diferentes fases de restauración (elección del sitio, de la especie, del método de propagación, etc.); protocolos probados en campo para la propagación y establecimiento de diferentes especies de Polylepis; mapas de distribución y guías de identificación de las especies de Polylepis en campo; difusión por medios digitales como blogs o revistas de difusión científica; e incluir en las publicaciones científicas las implicaciones que los resultados tienen para la práctica de la restauración. Un primer paso que podemos tomar los investigadores para que nuestros resultados sean más accesibles a los practicantes es tratar de publicar más de nuestros artículos científicos en castellano. A la vez, podríamos ayudar a los practicantes a comunicar los resultados que generen, 
brindándoles apoyo para publicarlos tanto en revistas científicas como en las de difusión técnica.

Una limitación considerable en muchos proyectos de reforestación es la falta de monitoreo una vez establecidas las plantas en campo. En algunos casos, esto se debe a la falta de capacidad técnica en el equipo de trabajo; en muchos otros, sin embargo, se debe a la falta de apoyo financiero para hacerlo (Le et al. 2012; Thomas et al. 2014). El sector académico podría contribuir realizando los monitoreos y así aprovechar estos proyectos como oportunidades para responder preguntas de investigación, y analizar la experiencia y los resultados. El monitoreo, el análisis de los resultados y su publicación permiten compartir las experiencias obtenidas con los demás interesados; además, permiten promover el manejo adaptativo y hacer ajustes dentro del mismo proyecto y equipo de trabajo.

Por último, aunque aquí solo tratamos aspectos biológicos en los que las ciencias naturales pueden acercarse a la práctica de la restauración, reconocemos que existen dimensiones sociales (e.g., valores culturales, económicos, políticos) que también afectan la sostenibilidad de estos esfuerzos (Egan et al. 2011). Los académicos ambientales pueden contribuir al conocimiento científico y evaluar las propuestas para enfrentar los aspectos sociales del manejo ambiental, incorporando en sus investigaciones conceptos y metodologías provenientes de las ciencias sociales (e.g., economía, sociología) o involucrando en las iniciativas de investigación a expertos de estas disciplinas (St. John et al. 2014). Como otros autores (e.g., Perring et al. 2015), sugerimos que la incorporación de otras disciplinas más allá de las ciencias naturales será clave para buscar respuestas integrales al conjunto de retos de restauración y manejo de los ecosistemas de Polylepis.

\section{Conclusiones}

En la actualidad, existe una brecha entre la ciencia y la práctica de la restauración de los bosques y arbustales dominados por Polylepis. Los académicos y los practicantes reconocemos prioridades diferentes, aunque a veces complementarias, en los vacíos de información ecológica que tenemos. Sin embargo, ambos sectores compartimos un objetivo común: encontrar soluciones que ayuden a superar barreras para generar proyectos de reforestación más sostenibles y que ayuden a recuperar estos ecosistemas clave. Para lograr esto es importante generar nuevo conocimiento científico, pero también mejorar el diálogo, fortalecer vínculos $\mathrm{y}$ promover colaboraciones reales entre académicos y practicantes.

Agradecimientos. Agradecemos a los organizadores del IV Congreso de Ecología y Conservación de Bosques de Polylepis por su apoyo logístico y servicio como anfitrión del simposio, con reconocimiento particular a G. Guzmán, y al UC Davis Plant Sciences Research Endowment que financió la cena de discusión en Jujuy, Argentina. También agradecemos los aportes de tres revisores anónimos al manuscrito final.

\section{Referencias}

Ashcroft, M. B., and J. R. Gollan. 2013. Moisture, thermal inertia, and the spatial distributions of near-surface soil and air temperatures: understanding factors that promote microrefugia. Agricultural and Forest Meteorology 176:77-89. DOI: 10.1016/j.agrformet.2013.03.008

Ayma-Romay, A. I., G. Soto-Rojas, and P. Lovera. 2015. Efecto de la altitud y el micrositio sobre plántulas reforestadas de Polylepis lanata (Rosaceae) en el noroeste de Cochabamba, Bolivia: implicaciones para su restauración ecológica. Ecología en Bolivia 50:7-24.

Cabin, R. J., A. Clewell, M. Ingram, T. McDonald, and V. Temperton. 2010. Bridging Restoration Science and Practice: Results and Analysis of a Survey from the 2009 Society for Ecological Restoration International Meeting. Restoration Ecology 18:783-788. DOI: 10.1111/j.1526-100X.2010.00743.x.

Cierjacks, A., S. Salgado, K. Wesche, and I. Hensen. 2008. Post-Fire Population Dynamics of Two Tree Species in HighAltitude Polylepis Forests of Central Ecuador. Biotropica 40:176-182. DOI: 10.1111/j.1744-7429.2007.00361.x.

Cuyckens, G. A. E., D. A. Christie, A. I. Domic, L. R. Malizia, and D. Renison. 2016. Climate change and the distribution and conservation of the world's highest elevation woodlands in the South American Altiplano. Global and Planetary Change 137:79-87. DOI: 10.1016/j.gloplacha.2015.12.010.

Egan, D., E. E. Hjerpe, and J. Abrams (eds.). 2011. Human Dimensions of Ecological Restoration: Integrating Science, Nature, and Culture. Island Press, Washington D.C., USA.

Falk, D. A., C. M. Richards, A. M. Montalvo, and E. E. Knapp. 2006. Population and Ecological Genetics in Restoration Ecology. Pp. 14-41 en D. A. Falk, M. A. Palmer and J. B. Zedler (eds.). Foundations of Restoration Ecology. Island Press, Washington D.C. USA. 
Fjeldsa, J. 2002. Polylepis forests-Vestiges of a vanishing ecosystem in the Andes. Ecotropica 8:111-123.

Fuentealba, B., and S. Sevillano. 2016. Experiencias de rehabilitación comunitaria con queñual (Polylepis sp.) en el Departamento de Ancash, Perú. Pp. 315-327 en E. Ceccon and D. Pérez (eds.). Más allá de la ecología de la restauración: perspectivas sociales en América Latina y el Caribe. $1^{a}$ edición. Vázquez Manzini Editores, Buenos Aires, Argentina.

Gareca, E. E., P. Breyne, K. Vandepitte, J. R. A. Cahill, M. Fernández, and O. Honnay. 2013. Genetic diversity of Andean Polylepis (Rosacea) woodlands and inferences regarding their fragmentation history. Botanical Journal of the Linnean Society 172:544-554. DOI: 10.1111/boj.12055.

Gareca, E. E., M. Hermy, J. Fjeldsa, and O. Honnay. 2010. Polylepis woodland remnants as biodiversity islands in the Bolivian high Andes. Biodiv Conserv 19:3327-3346. DOI: 10.1007/s10531-010-9895-9.

Gareca, E. E., Y. Y. Martínez, R. O. Bustamante, L. F. Aguirre, and M. M. Siles. 2007. Regeneration patterns of Polylepis subtusalbida growing with the exotic trees Pinus radiata and Eucalyptus globulus at Parque Nacional Tunari, Bolivia. Plant Ecology 193:253-263. DOI: 10.1007/s11258-007-9263-3.

Hensen, I., A. Cierjacks, H. Hirsch, M. Kessler, K. Romoleroux, D. Renison, and K. Wesche. 2012. Historic and recent fragmentation coupled with altitude affect the genetic population structure of one of the world's highest tropical tree line species. Global Ecology and Biogeography 21:455-464. DOI: 10.1111/j.1466-8238.2011.00691.x.

Hensen, I., I. Teich, H. Hirsch, H. von Wehrden, and D. Renison. 2011. Range-wide genetic structure and diversity of the endemic tree line species Polylepis australis (Rosaceae) in Argentina. American Journal of Botany 98:1825-1833. DOI: 10.3732 /ajb.1000100.

Kessler, M. 2002. The "Polylepis problem": Where do we stand? Ecotropica 8:97-110.

Kessler, M. 2006. Bosques de Polylepis. Páginas 110-120 en M. Moraes, B. Øllgard, L. P. Kvist, F. Borchsenius and H. Balslev (eds.). Bótanica Económica de los Andes Centrales. Universidad Mayor de San Andrés, La Paz, Bolivia.

Kessler, M., J. M. Toivonen, S. P. Sylvester, J. Kluge, and D. Hertel. 2014. Elevational patterns of Polylepis tree height (Rosaceae) in the high Andes of Peru: role of human impact and climatic conditions. Frontiers in Plant Science 5: 1-12. DOI: $10.3389 /$ fpls.2014.00194.

Le, H. D., C. Smith, J. Herbohn, and S. Harrison. 2012. More than just trees: assessing reforestation success in tropical developing countries. Journal of Rural Studies 28:5-19. DOI: 10.1016/j.jrurstud.2011.07.006.

Mindreau, M., and C. Zuñiga. 2010. Manual de Forestería Comunitaria de Alta Montaña: Experiencias de Reforestación con Polylepis sp. en el Corredor de Conchucos-Ancash. Instituto de Montaña, Huaraz, Perú.

Morales, L. V. 2017. Polylepis Regeneration and the Potential for Forest Expansion in the Peruvian Andes: The Influence of Cattle and Environmental Conditions. PhD Thesis. Ecology, University of California, Davis, California. U.S.A. Pp.110.

Navarro, G., S. Arrázola, J. A. Balderrama, W. Ferreira, N. De la Barrra, C. Antezana, I. Gómez, and M. Mercado. 2010. Diagnóstico del estado de conservación y caracterización de los bosques de Polylepis en Bolivia y su avifauna. Rev Bol Ecol y Cons Amb 28:1-35.

Orrego, F. 2011. Queñoa: Árbol de las alturas. Gerencia de Medio Ambiente de la Compañía Minera Doña Inés de Collahuasi, Santiago, Chile.

Palmer, M. A. 2009. Reforming Watershed Restoration: Science in Need of Application and Applications in Need of Science. Estuaries and Coasts 32:1-17. DOI: 10.1007/s12237-008-9129-5.

Peng, Y., L. Morales, I. Hensen, and D. Renison. 2017. No effect of elevation and fragmentation on genetic diversity and structure in Polylepis australis trees from central Argentina. Austral Ecology 42:288-296. DOI: 10.1111/aec.12432.

Perring, M. P., R. J. Standish, J. N. Price, M. D. Craig, T. E. Erickson, K. X. Ruthrof, A. S. Whiteley, L. E. Valentine, and R. J. Hobbs. 2015. Advances in restoration ecology: rising to the challenges of the coming decades. Ecosphere 6:131. DOI: 10.1890/ES15-00121.1.

Purcell, J., and A. Brelsford. 2004. Reassessing the causes of decline of Polylepis, a tropical subalpine forest. Ecotropica 10:155-158.

Rada, F., C. García-Núñez, and S. Rangel. 2009. Low temperature resistance in saplings and ramets of Polylepis sericea in the Venezuelan Andes. Acta Oecologica 35:610-613. DOI: 10.1016/j.actao.2009.05.009.

Rada, F., C. García-Núñez, and S. Rangel. 2011. Microclimate and Regeneration Patterns of Polylepis sericea in a treeline forest of the Venezuelan Andes. Ecotrópicos 24:113-122.

Rangel, J. O., and H. Arellano. 2010. Bosques de Polylepis: Un tipo de vegetación condenado a la extinción. Pp. 443-477 en J. O. Rangel-CH(ed.). Colombia Diversidad Biótica X. Cambio global (natural) y climático (antrópico) en el páramo colombiano. Universidad Nacional de Colombia, Instituto de Ciencias Naturales, Bogotá D.C., Colombia.

Renison, D., and A. M. Cingolani.1998. Experiencias en germinación y reproducción vegetativa aplicados a la reforestación con Polylepis australis (Rosaceae) en las Sierras Grandes de Córdoba, Argentina. AgriScientia 15:47-53.

Renison, D., A. Cingolani, R. Suárez, E. Menoyo, C. Coutsiers, A. Sobral, and I. Hensen. 2005. The Restoration of Degraded Mountain Woodlands: Effects of Seed Provenance on Polylepis australis Seedling Survival and Growth in Argentina. Restoration Ecology 13:129-137. DOI: 10.1111/j.1526-100X.2005.00015.x.

Renison, D., M. P. Chartier, M. Menghi, P. I. Marcora, R. C. Torres, M. Giorgis, I. Hensen, and A. M. Cingolani. 2015. Spatial variation in tree demography associated to domestic herbivores and topography: Insights from a seeding and planting experiment. Forest Ecology and Management 335:139-146. DOI: 10.1016/j.foreco.2014.09.036.

Renison, D., G. A. E. Cuyckens, S. Pacheco, G. F. Guzmán, H. R. Grau, P. Marcora, G. Robledo, A. M. Cingolani, J. Domínguez, M. Landi, L. Bellis, and I. Hensen. 2013. Distribución y estado de conservación de las poblaciones de 
árboles y arbustos del género Polylepis (Rosaceae) en las montañas de Argentina. Ecología Austral 23: 27-36.

Renison, D., M. L. Herrero, R. C. Torres, R. Suárez, P. Friedlander, S. E. Navarro Ramos, F. Barri, and A. M. Cingolani. 2016. El rol de los voluntariados en la restauración ecológica del centro argentino. Pp. 55-76 en E. Ceccon and D. Pérez (eds.). Más allá de la ecología de la restauración: perspectivas sociales en América Latina y el Caribe. $1^{a}$ edición. Vázquez Manzini Editores, Buenos Aires, Argentina.

Renison, D., L. V. Morales, E. Cuyckens, C. S. Sevillano, and D. M. Cabrera Amaya. Este número. Ecología y conservación de los bosques y arbustales de Polylepis: ¿qué sabemos y qué ignoramos? Ecología Austral X:XX-XX.

Reynel, C., and J., Marcelo. 2009. Quinual (Polylepis racemosa). Páginas 133-139 en Árboles de los ecosistemas forestales andinos. Manual de identificación de especies. Serie Investigación y Sistematización No. 9. Programa Regional ECOBONA-INTERCOOPERATION, Lima, Perú.

Rhymer, J. M., and D. Simberloff. 1996. Extinction by hybridization and introgression. Annual Review of Ecology and Systematics 27:832-109.

Rudnick, D., S. Ryan, P. Beier, S. A. Cushman, F. Diffenbach, C. W. Epps, L. R. Gerber, J. Harterr, J. S. Jenness, J. Kintsch, A. M. Merenlender, R. M. Perkl, D. V. Preziosi, and S. C. Trombulak. 2012. The role of landscape connectivity in planning and implementing conservation and restoration priorities. Issues in Ecology 16:1-20.

Sarango, A. Y. Modelo de Gestión Forestal para el DMQ. Octubre 2013. Secretaría del Ambiente, Quito, Ecuador. Secretaría de Ambiente, Quito, Ecuador. URL:https://goo.gl/5kLvHW.

Schmidt-Lebuhn, A. N., M. Kessler, and M. Kumar. 2006. Promiscuity in the Andes: Species Relationships in Polylepis (Rosacea, Sanguisorbea) Based on AFLP and Morphology. Systematic Botany 31:547-559. DOI: 10.1016/ j.flora.2005.07.007.

Simpson, B. B. 1979. A Revision of the Genus Polylepis (Rosacea: Sanguisorbeae). Smithsonian Contributions to Botany 43:1-62.

Segovia-Salcedo, M. C. 2011. Los riesgos de la reforestación de los páramos con especies exóticas: el caso de Polylepis racemosa. Propuestas Andinas 4(1).

Segovia-Salcedo, M., P. Quijia, K. Proaño-Tuma, D. Soltis, and P. Soltis. 2011. El estado de conservación de los bosques de yagual en el Ecuador (Polylepis, Rosacea: Rosoidea: Sanguisorbea): hibridacíon, translocación e introducción de especies exóticas. Serie Páramo 28:51-61.

Segovia-Salcedo M. C., and P. Quijia-Lamiña. 2014. Citogeografía de cuatro especies de Polylepis (Rosacea) en el Ecuador: Información relevante para el manejo y conservación de los bosques andinos. Pp. 467-485 en F. Cuesta, J. Sevink, L. D. Llambi, B. De Bievre and J. Posner (eds.). Avances en investigación para la conservación de los páramos andinos. CONDESAN.

Seltmann, P., I. Hensen, D. Renison, K. Wesche, S. Ploch, J. Rondan Dueñas, A. Cocucci, and K. Jung. 2009. Biparental inbreeding depression, genetic relatedness, and progeny vigour in a wind-pollinated treeline species in Argentina. Plant Ecology 205:155-164. DOI: 10.1007/s11258-009-9605-4.

Society for Ecological Restoration (SER) International. 2004. Principios de SER International sobre la restauración ecológica. Society for Ecological Restoration International, grupo de trabajo sobre ciencia y políticas. URL: Www. ser.org. Tucson, Arizona, U.S.A.

Sevillano-Ríos, C. S., L. V. Morales, and A. D. Rodewald. Este número. Ecología y conservación de las aves asociadas con Polylepis: ¿qué sabemos de esta comunidad cada vez más vulnerable? Ecología Austral X:XX-XX.

St John, F. A. V., A. M. Keane, J. P. G. Jones, and E. J. Milner-Gulland. 2014. Robust study design is as important on the social as it is on the ecological side of applied ecological research. Journal of Applied Ecology 51:1479-1485. DOI: 10.1111/1365-2664.12352.

Thomas, E., R. Jalonen, J. Loo, D. Boshier, L. Gallo, S. Cavers, S. Bordacs, P. Smith, and M. Bozzano. 2014. Genetic consideration in ecosystem restoration using native tree species. Forest Ecology and Management 333:66-75. DOI: 10.1016/j.foreco.2014.07.015.

Torres, R. C., D. Renison, I. Hensen, and R. Suárez. 2008. Polylepis australis' regeneration niche in relation to seed dispersal, site characteristics and livestock density. Forest Ecology and Management 254:255-260. DOI: 10.1016/ j.foreco.2007.08.007. 